\title{
Driving Performance Experimental Analysis of Series Chopper Based EV Power Train
}

\author{
Atsuo Kawamura*, Giuseppe Guidi*, Yuki Watanabe ${ }^{* *}$, Yukinori Tsuruta*, Naoki Motoi*, \\ and Tae-Woong $\mathrm{Kim}^{\dagger}$ \\ *Yokohama National University, Yokohama, Japan \\ ${ }^{* *}$ Denso Corporation, Aichi, Japan \\ †Dept. of Control and Instrumentation Eng., Gyeongsang National University, Jinju, Korea
}

\begin{abstract}
In this paper, the series chopper based power train for electric vehicle is proposed for aiming the increase of one battery charge driving distance. Both the motor test bench based experiments and the chassis dynamo based experiments are tested and then analyzed for EV driving performance that the proposed power train with SAZZ chopper and Quasi-PAM control scheme is compared with or without the series chopper. It is confirmed through two kinds of experimental analyses that the series chopper power train for EV can be useful to extend the driving distance if the high efficiency and the low weight of chopper are satisfied.
\end{abstract}

Keywords:Drivingperformance, Electric vehicle, PAM, PWM, Quasi-PAM inverter, SAZZ chopper

\section{INTRODUCTION}

The use of EV (Electric Vehicle) instead of internal combustion engine vehicle can expect to save the total energy consumption under friendly environments[1]. The ultimate goal of such a vehicle is to drive a longer and longer distance under any driving patterns. At the present, taking the battery storage capability technology and the cost into consideration, it has been reported that $\mathrm{EV}$ is suitable to the urban drive including a short driving distance commuter[2], [3].

From the view point of practical EV use in city areas, the urban driving pattern such as JC08 mode in Japan or LA-4 mode in USA gives a prediction of actual driving range in a city areas, thus the measurement regulation is strictly controlled by each country's laws[4]. Under such driving modes, the total driving distance of $\mathrm{EV}$ becomes the main performance function with each component's efficiency, battery characteristics, and total system design. In total system design, the power train system design itself for EV can also give a large influence to the total driving range. The power train system for EV drive is basically consisting of battery and inverter. If necessary, chopper is inserted between battery and

Manuscript received Jan. 26, 2012; revised Oct.16, 2012

Recommended for publication by Associate Editor Jin Hur.

${ }^{\dagger}$ CorrespondingAuthor:kimtw@gnu.ac.kr

Tel: +82-55-772-1746, Gyeongsang National University

*Yokohama National University, Japan

Denso Corporation, Japan

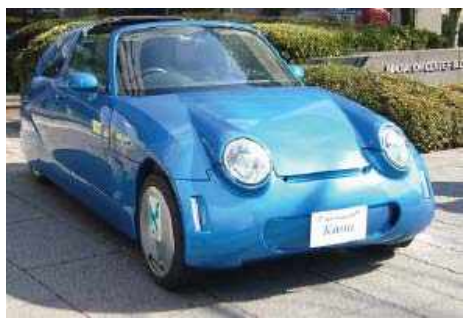

(a) front view

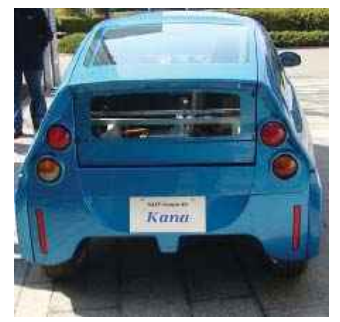

(b) rear view.
Fig. 1. Appearance of KAST-EV "KANA".

inverter. Thus, each component itself for $\mathrm{EV}$ power train system requires a high efficiency regardless of driving pattern to drive a longer and longer distance under the given battery charges.

Taking the descriptions mentioned above into consideration, authors have proposed that the series SAZZ (Snubber Assisted Zero voltage and Zero current transition) chopper based power train for EV has advantages over the chopperless based power train. The SAZZ chopper[5]-[11] has been improved over $98 \%$ in efficiency and the Qausi-PAM control scheme of inverter[15] has been also developed to reduce the switching losses. In addition, the prototype electric vehicle has been developed for EV performance evaluation and its appearance is shown in Fig. 1, which is called KAST-EV[13], [14]. It will be verified by two kinds of experimental analysis such as the motor test bench in the laboratory and the chassis dynamo in the driving performance evaluation facility that the proposed 
EV power train with SAZZ chopper and Quasi-PAM control scheme is better in the total driving performance evaluations.

In this paper, advantage of series chopper based EV power train will be in detail described in the chapter II, and in the chapter III the total efficiency analysis for EV will be presented. As a result, SAZZ chopper which have been developed by authors may give a solution for EV optimization. Driving performance analysis of the proposed EV power train will be given by two types of experiments in chapter IV and V. Finally the chapter VI will make a conclusion of this paper.

\section{ELECTRIC VehicLE PoWer TRAINS}

Fig. 2 shows two types of electric vehicle power trains.

As shown in Fig. 2(a), the typically and widely used power train for EV is basically a battery-inverter-motor combination. Battery DC voltage $V_{\text {batt }}$ is converted to the required 3 phase voltage $V_{\mathrm{abc}}$ by inverter for motor drives. If two in-wheel motors are employed for EV, two inverters are generally required. This kind of power train for $\mathrm{EV}$ is called "non-chopper type" or "chopperless type" conventional power train in this paper. This power train has advantages in simplicity and well-established technology. However, from the view point of energy saving, it has disadvantages. For example, at the lower speed region, PWM (Pulse Width Modulation) pulses of inverter become very narrow. As a result, the efficiency of EV can be lower compared with chopper based power train, which can be functioned as PAM (Pulse Amplitude Modulation) typed voltage regulation. And also, at the high speed region, the higher $\mathrm{AC}$ voltage is required for motor drive. Thus, the EV power train should be taken into consideration for improving driving performance such as longer driving distance. Because the battery DC voltage is varied during operation, both motor and inverter should be optimally designed under the given EV's specifications to keep a higher efficiency. Furthermore, the battery voltage drop at the low SOC (State of Charge) may be compensated by the voltage boost system for higher efficiency, compactness, and lighter weight.

To solve the problems mentioned above, authors have been proposing the series SAZZ chopper based power train for EV and FCEV (Fuel Cell Electric Vehicle)[4] in Fig. 2(b).

\section{A. Series Chopper Based EV Power Train}

To design the proposed power train for EV, DC-DC chopper should meet the followings: (a) high efficiency, (b) compactness, (c) light weight, and (d) high reliability.

SAZZ chopper, developed by authors, is one candidate as the suitable DC-DC chopper because SAZZ chopper has a high efficiency and a high reliability by implementing soft switching technology. In [11], 98\% efficiency of SAZZ was reported under bi-directional 2 quadrant operation(buck and

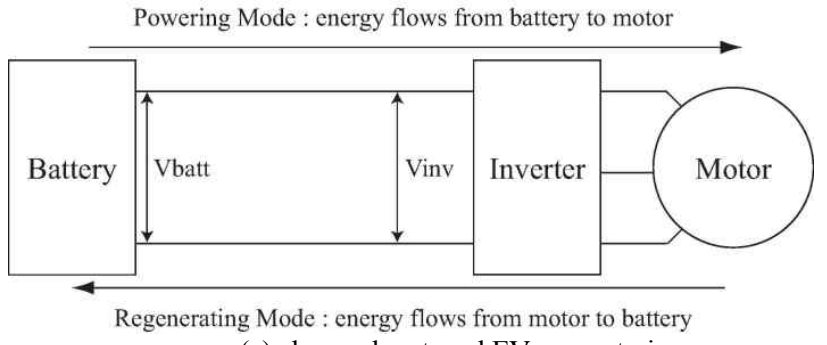

(a) chopperless typed EV power train.

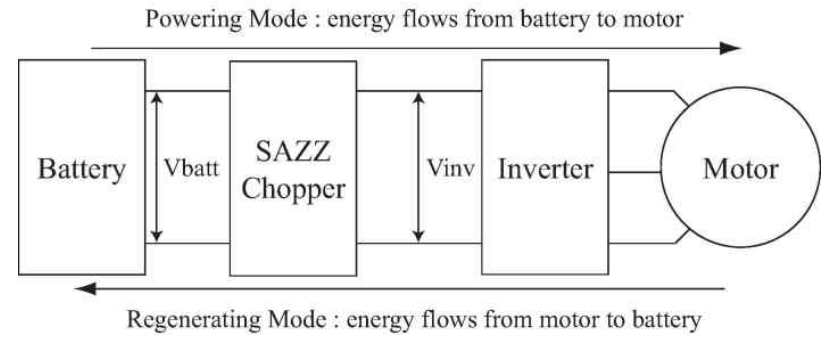

(b) series chopper typed EV power train.

Fig. 2. Types of electric vehicle power trains: $V_{\text {inv }}$ in (a) is fixed by $V_{\text {batt }}$, but $V_{\text {abc }}$ in (b) is controlled by chopper from $V_{\text {batt }}$.

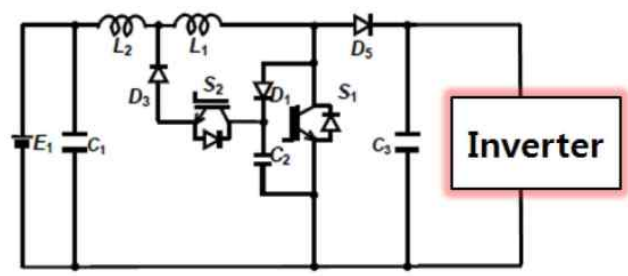

(a) basic circuit of boost SAZZ chopper.

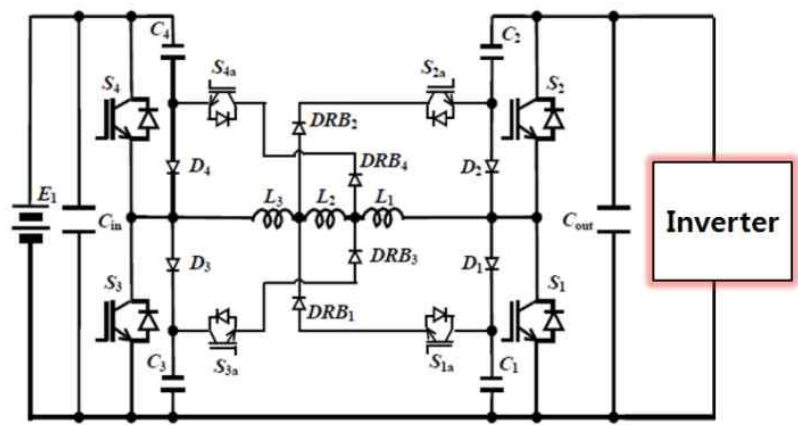

(b) two quadrant circuit of bi-directional boost/buck SAZZ chopper.

Fig. 3. SAZZ chopper: (a) basic topology, (b) bi-2Q topology for KAST-EV.

boost functions in case of both powering and regenerating modes) and $28 \mathrm{~kW}$ output power. And a very high power density of about $30 \mathrm{~kW} / l$ was also reported in [10]. The SAZZ chopper is shown in Fig. 3: (a) is for basic configuration circuit of SAZZ chopper and (b) is for bi-directional boost/buck SAZZ chopper which is installed in the prototype EV "KAST-EV". 
TABLE I

Classified Modulation SCHEMES For INVERTER DRive

\begin{tabular}{c|l}
\hline scheme & \multicolumn{1}{c}{ pulse pattern and characteristics } \\
\hline \hline PWM & modulated only pulse width (Pure-PWM) \\
\hline PAM & modulated only pulse amplitude (Pure-PAM) \\
\hline Quasi-PAM & $\begin{array}{l}\text { modulated both pulse amplitude and pulse width } \\
\text { modulation combined by both PWM and PAM } \\
\text { - natural modulation based on clamped ref. voltage } \\
\text { - one wide pulse at the center and pulse trains at both } \\
\text { sides }\end{array}$ \\
\hline
\end{tabular}

Chopper can be designed for EV drive as either unidirectional 2 quadrant operation (uni-2Q for abbreviation) or bidirectional 2 quadrant operation (bi-2Q for abbreviation). A typical uni-2Q chopper is applied to Toyota brand vehicle "Prius", in which a hard switching 2Q chopper (boost function in the case of powering mode quadrant operation and buck function in the case of regenerating mode quadrant operation) is installed[12]. If bi-2Q operation chopper is designed for EV[9], the inverter-motor can be more flexibly designed and then the overall system for EV drive can be optimally designed.

Under no-load torque conditions, the terminal voltage $V_{\text {batt }}$ of Lithium-ion battery is changed from $100 \%$ down to $60 \%$ of the rated voltage as SOC is changed from $100 \%$ to $0 \%$. If bi-2Q chopper is used as DC-DC power conversion for EV drive, it has advantages that powering-regenerating operation in the low speed region may be achieved at the higher efficiency compared with that of uni-2Q chopper. The DC-DC power conversion loss of bi-2Q chopper is larger than that of uni-2Q chopper, because the number of the series switch is one in uni-2Q chopper and two in bi-2Q chopper.

\section{B. Modulation Schemes for Inverter Drive}

When chopper is installed between battery and inverter, the input DC voltage $V_{\text {inv }}$ of inverter can be easily controllable. PAM scheme for inverter drive can improve the EV system efficiency. Fig. 4 shows pulse waveforms generated by PWM scheme and PAM scheme for inverter drive. As shown in TABLE I, the modulation schemes for inverter drive are classified in this paper.

PAM inverter for EV drive has merits and demerits in efficiency improvement and system performance. The loss of inverter includes switching loss, conduction loss, and leakage current loss[15],[16]: the switching loss is almost proportional to the switching frequency of inverter, the conduction loss is almost the same between PAM inverter and PWM inverter, and the leakage current loss is negligible. Generally, the loss of motor has a tendency of loss increase when THD (Total Harmonic Distortion) of inverter output voltage $V_{\mathrm{abc}}$ increases. However, if the input DC voltage $V_{\text {inv }}$ of inverter is controlled by DC-DC power conversion system according to the conditions of speed and load torque,

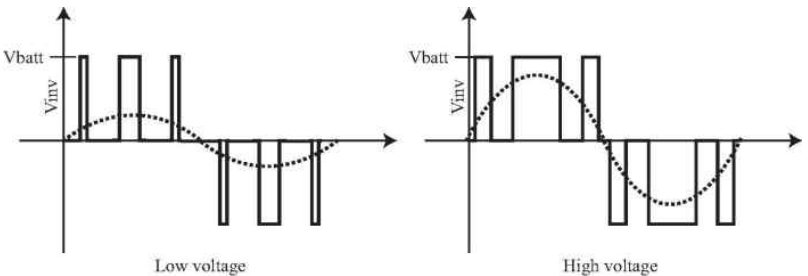

(a) PWM pulse waveform (Pure-PWM).

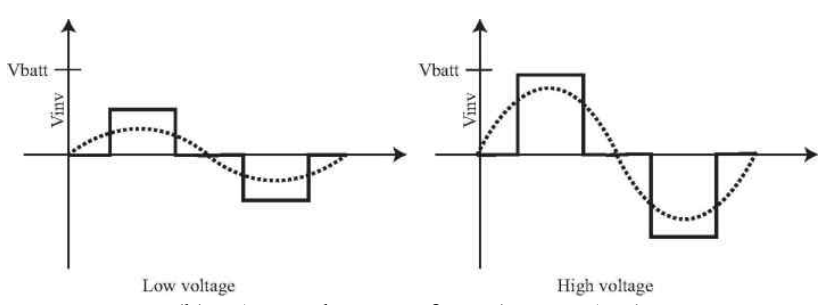

(b) PAM pulse waveform (Pure-PAM).

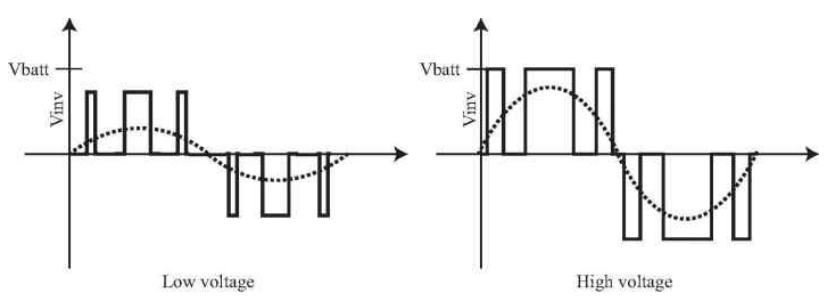

(c) Quasi-PAM pulse waveform.

Fig. 4. Pulse waveforms generated by modulation schemes of inverter: $V_{\text {inv }}$ in (a) is fixed by $V_{\text {batt, }}$, but $V_{\text {inv }}$ in (b) and (c) can be controlled lower or larger than $V_{\text {batt }}$ by chopper.

the efficiency of motor can be improved.

Motor efficiency is dependent on absolute value of inverter output harmonic voltages, thus the efficiency of the motor largely depends on the pulse pattern selected according to the conditions of speed and load torque.

\section{EFFICIENCY IMPROVEMENT METHODS OF EV}

The basic concept for EV drive is illustrated in Fig. 2 and Fig. 4. Thus, the concrete implementation of each component will be discussed in this section from the practical viewpoints of efficiency improvement.

\section{A. Implementation of Series Chopper Based EV Power Train}

When the experimental system with the proposed EV power train is set up according to Fig. 2(b), two types of PAM controlled inverters and one pure PWM controlled inverter can be implemented. The modulation schemes and their characteristics of inverter were in detail discussed in the previous subsection II-B.

One is the general PAM controlled inverter, which both phase and amplitude of output voltage for PMSM drive are independently controlled by vector controlled inverter and the input DC voltage of inverter is quickly and precisely 
controlled by the series chopper according to the reference amplitude of the input DC voltage. In other words, only pulse amplitude is controlled under keeping the same pulse width. Fig. 4(b) shows that there is only one pulse at the half periodic center of line voltage, in which the amplitude is controlled by chopper.

Another approach is the Quasi-PAM controlled inverter, which is similar to the general PAM controlled inverter except that the suitable pulse trains at both sides are partially modulated by PWM scheme. The Quasi-PAM controlled inverter can generate the suitable pulse pattern by both the naturally modulated pulse amplitude at center and the modulated pulse widths at both sides. The pulse pattern by Quasi-PAM inverter is shown in Fig. 4 (c). In Quasi-PAM controlled inverter, the input DC voltage $V_{\mathrm{inv}}$ of inverter is controlled by chopper to be a little larger than the induced motor voltage depending on the conditions of PMSM speed and load torque.

The other approach is the pure PWM controlled inverter under the constant input DC voltage, as shown in Fig. 4(a). As the PWM controlled inverter has been well known, there are no additional explanations and discussions in this subsection.

In this paper, the experimental analysis will be performed on the basis of two kinds of modulation schemes: one is the Quasi-PAM controlled inverter with SAZZ chopper (Quasi-PAM for abbreviation) and the other is the PWM controlled inverter without chopper (Pure-PWM for abbreviation).

\section{B. Loss Evaluations of EV Power Train}

As mentioned above, the power train for EV drive is consisting of 4 basic components: (a) battery, (b) chopper, (c) inverter, and (d) electric motor such as PMSM. In advance, sorts of power train losses will be explained and then the experimental data obtained from the motor test bench and the chassis dynamo will be in detail analyzed in the next two sections of IV and V.

\section{1) Inverter Loss}

Inverter loss is mainly composed of the switching loss $P_{\mathrm{sw}}$ and the conduction loss $P_{\text {on }}$, which are respectively expressed as the following equations[16].

$$
\begin{gathered}
P_{\mathrm{sw}}=\frac{1}{6} \times V_{\mathrm{inv}} \times I_{\mathrm{ave}} \times\left(\Delta T_{\mathrm{r}}+\Delta T_{\mathrm{f}}\right) \times f_{\mathrm{sw}} \\
P_{\mathrm{on}}=V_{\text {on }} \times I_{\text {ave }} \times d
\end{gathered}
$$

where $V_{\mathrm{inv}}$ is input DC voltage of inverter, $I_{\mathrm{ave}}$ is average current, $V_{\mathrm{on}}$ is on-voltage of switch, $\Delta T_{\mathrm{r}}$ and $\Delta T_{\mathrm{f}}$ are turn-on rising time and turn-off falling time respectively, $f_{\mathrm{sw}}$ is switching frequency of inverter, and $d$ is duty ratio.
Through (1) and (2), it is firmly confirmed that switching loss is largely proportional to $V_{\text {inv }}$ and $f_{\text {sw }}$ and conduction loss is dependent on on-voltage of switch.

As shown in Fig. 4, $V_{\text {inv }}$ can be lower in a low speed region with PAM control scheme, while $V_{\text {inv }}$ is fixed to the high battery voltage $V_{\text {batt }}$ in the PWM control scheme. Therefore, as the PAM or Quasi-PAM controlled inverter is introduced, the inverter efficiency may be increased by changing the input DC voltage $V_{\mathrm{inv}}$ of inverter. In this paper, Quasi-PAM controlled inverter is appropriately adopted according to speed region to reduce switching loss while the output voltage harmonics is optimally suppressed.

\section{2) Motor Loss}

Regarding motor loss, there are copper loss and iron loss. Furthermore, iron loss of motor is composed of hysteresis loss and eddy current loss. Under the applied sinusoidal voltage to motor, hysteresis loss $P_{\mathrm{h}}$ and eddy current loss $P_{\mathrm{e}}$ for iron losses are respectively expressed in the following equations[17],[18],[22]-[24].

$$
\begin{aligned}
& P_{\mathrm{h}}=k_{\mathrm{h}} \times \mathrm{f} \times B_{m}^{\beta} \\
& P_{\mathrm{e}}=k_{\mathrm{e}} \times f^{2} \times B_{m}^{2}
\end{aligned}
$$

where $k_{\mathrm{h}}$ and $k_{\mathrm{e}}$ are the coefficients of hysteresis loss and eddy current loss respectively, $f$ is the output voltage frequency, $B_{m}$ is the maximum flux density, and $\beta$ is Steinmetz's constant $(=1.6 \sim 2)$ which is determined by experiments.

The maximum flux density $B_{m}$ has the following relations.

$$
B_{m}=k_{m} \times \frac{V_{m}}{S \cdot f}
$$

where $k_{m}$ is the coefficient of flux density, $V_{m}\left(=V_{\mathrm{abc}}\right)$ is the applied voltage to motor, and $S$ is the cross section area $\left[\mathrm{m}^{2}\right]$ of iron.

Putting (5) into (3) and (4) respectively, the hysteresis loss and the eddy current loss can be rewritten as the following equations, which are related to flux density.

$$
\begin{aligned}
& P_{\mathrm{h}}=\frac{k_{\mathrm{h}} \cdot k_{m}^{\beta}}{S^{\beta}} \times \frac{V_{m}^{\beta}}{f^{\beta-1}}=\frac{k_{1}}{S^{\beta}} \times \frac{V_{m}^{\beta}}{f^{\beta-1}} \\
& P_{\mathrm{e}}=\frac{k_{\mathrm{e}} \cdot k_{m}^{2}}{S^{2}} \times V_{m}^{2}=\frac{k_{2}}{S^{2}} \times V_{m}^{2}
\end{aligned}
$$

where $k_{1}=k_{\mathrm{h}} \cdot k_{m}^{\beta}$ and $k_{2}=k_{\mathrm{e}} \cdot k_{m}^{2}$.

Through (6) and (7), it can be predicted that the iron loss is proportional to the applied voltage pulse amplitude to motor. The motor voltage amplitude by Quasi-PAM inverter can be less than that by pure PWM inverter. Therefore, the motor efficiency can be improved due to the iron loss reduction if Quasi-PAM inverter is used.

Under the applied harmonic voltage to motor, the hysteresis loss $P_{h, n}$ and the eddy current loss $P_{e, n}$ for 
harmonic iron losses are respectively expressed in the following equations[22]-[24]. The total hysteresis loss and the eddy current loss are calculated by the sum of the harmonic losses as follows:

$$
\begin{aligned}
& P_{h, n}=\int_{\text {iron }} \sum_{n}^{M} k_{\mathrm{h}} D(n f)^{2}\left\{B_{r, n}^{2}+B_{\theta, n}^{2}\right\} d v \\
& P_{e, n}=\int_{\text {iron }} \sum_{n}^{M} k_{\mathrm{e}} D(n f)^{2}\left\{B_{r, n}^{2}+B_{\theta, n}^{2}\right\} d v
\end{aligned}
$$

where $n$ is the harmonic order, $M$ is the number of harmonics, $D$ is the density of core, and $B_{r, n}$ and $B_{\theta, n}$ are the $n$th harmonics of the radial and peripheral components of flux density respectively.

On the basis of iron losses mentioned above, the total iron losses of motor can be expressed as follows:

$$
\begin{aligned}
& P_{\text {total iron }}= \\
& P_{\text {iron }, 1}\left(\alpha, B_{\max }\right)+\sum_{n=2}^{M}\left\{P_{\text {iron, } \mathrm{n}}\left(B_{r, n}\right)+P_{\text {iron, } \mathrm{n}}\left(B_{\theta, n}\right)\right\}
\end{aligned}
$$

where $P_{\text {iron, } 1}$ and $P_{\text {iron, n }}$ are the fundamental and the harmonic iron losses respectively, and $\alpha$ is the axis ratio of the rotating magnetic flux defined by $B_{\min } / B_{\max }$.

Even though Quasi-PAM inverter is used for loss reduction, harmonic iron loss can't be neglected because Quasi-PAM inverter makes harmonics a little larger than Pure-PWM inverter. However it can be expected that the switching loss is much reduced from the inverter viewpoints, compared to influence of motor harmonic iron loss.

\section{3) Review of Losses under Regenerating Operation}

To improve one charge driving distance longer, the regenerating operation in EV drive is another important factor to maximize the regenerating energy. The regenerating operation is, to some extent, similar to the powering operations mentioned above. Only the differences in both operations are the direction of energy flow and the slightly different amount of conduction loss.

Regardless of powering mode or regenerating mode in EV drives, the total loss by Quasi-PAM inverter can be smaller than that by Pure-PWM inverter. The conduction loss of inverter may be a slight difference between powering operation and regenerating operation. The current flows in free-wheeling diodes has the longer period in the regeneration mode than that in powering mode, and then the on-state voltage of switching devices such as IGBTs and diodes makes a different conduction loss.

\section{4) Battery Characteristics and Inverter-Motor Design}

The equivalent circuit of a battery is a combination of an internal resistance and a variable voltage source[19],[20]. The battery voltage decreases non-linearly as SOC decreases. The internal resistance should be smaller from the view point of the total energy management.
However, the DC battery energy is converted to AC electricity by an inverter for applying to a motor. When the motor speed or the vehicle speed becomes high, the CEMF (Counter Electro Motive Force) voltage of the motor also increases. Thus, in general, the inverter output voltage $V_{\mathrm{abc}}$ is almost proportional to the rotor speed. Otherwise the required electric torque cannot be produced in the high speed region under the given load torque condition. If DC-DC chopper is inserted between battery and inverter, the input DC voltage $V_{\text {inv }}$ of inverter can be controlled even though the battery voltage $V_{\text {batt }}$ decreases due to SOC's decrease. In case of no DC-DC power conversion system, the motor terminal voltage $V_{m}$ should be specified to the lowest battery voltage under the maximum rotor speed. The detail motor design is not simple to discuss here in case that the applied motor terminal voltage is changed. However, the brief discussion regarding efficiency is as follows.

Let us compare two kinds of motor design "Type A" and "Type B" under the following assumptions. (1) The maximum rotor speed, the output power at the maximum speed, and the pole number are the same in both Type A and Type B. (2) The applied rms voltage of Type A is 1pu (per unit) at the maximum speed and that of Type B is $0.5 p u$. Since the maximum speed and the output power at the maximum speed are the same, the Ampere-turns of the windings in both motors should be the same. If Type A has \$1puwinding turns, Type B should have 0.5puwinding turns. Therefore if the current of Type A is $I[\mathrm{pu}]$, that of Type B should be $2 I[\mathrm{pu}]$.

If the motor size is the same between two types, and the slot cross section is the same. Therefore if the total resistance of Type A winding is expressed as $r[\mathrm{pu}]$, that of Type B becomes $0.25 r[\mathrm{pu}]$. Because it is assumed that the winding length becomes half and the winding cross section becomes 2 times larger. If the joule loss of Type A is $r \times I^{2}[\mathrm{pu}]$, that of Type $\mathrm{B}$ is $0.25 r \times(2 I)^{2}=r \times I^{2}[\mathrm{pu}]$.

This simple discussion can say that the motor copper loss with or without input DC voltage control for inverter does not be changed in principle, if the same inverter frequency and the output power at the maximum speed under the same pole number are assumed.

The extra length of the winding in Type B can become a cause of the new loss, thus the motor efficiency of Type B may be a little lower than that of Type A. Of course, the inverter efficiency of Type A is higher than that of Type B because the output current through inverter is 2 times different.

Authors believe that, from the view point of the total efficiency improvement, the higher DC voltage of inverter has inherently advantages over the lower DC voltage. But this is not easy to prove experimentally, because two sets of total system should be compared. 
On the other hand, the Quasi-PAM controlled inverter and the Pure-PWM controlled inverter can be compared experimentally under the same inverter-motor set. The inverter-motor design is not changed from the initial design. However, the difference of two systems appears when the battery voltage $V_{\text {batt }}$ decreases as SOC decreases. Without chopper, the motor cannot achieve the maximum speed operation because of the insufficient CEMF voltage from inverter. The field weakening control is one technique to realize the high speed operation[17], but the efficiency of the motor drops substantially in this method. And the motor design which allows a wide speed range operation of field weakening makes the total efficiency lower. On the contrary, the Quasi-PAM controlled inverter can run the electric vehicle until the maximum speed because the input DC voltage $V_{\mathrm{inv}}$ of inverter is controlled at any value. For this reason, in the high speed region, the Quasi-PAM controlled controller shows the better performance than Pure-PWM controlled inverter.

\section{5) Efficiency of Series Chopper}

The total efficiency in EV drives is the multiplication of DC-DC chopper efficiency. Thus, chopper should have a high efficiency, a light weight, and a compact volume. Authors have been proposing the soft switching chopper, called SAZZ chopper past few years, which is suitable for fuel cell and/or electric vehicles. The details regarding SAZZ chopper can be found in the literatures[7], [9], [10]. The SAZZ chopper with 4 quadrant operation has the efficiency $98 \%$ under the output power $25 \mathrm{~kW}$, and the prototype in the laboratory has the power density of about $30 \mathrm{~kW} / \mathrm{l}$.

\section{Motor Test Bench BAsed DRIVING PERFORMANCE EXPERIMENTAL ANALYSIS}

\section{A. Configuration of Motor Test Bench}

In order to verify the proposed concept, the experimental set-up is constructed in the laboratory, which is called "motor-test-bench". The overall schematic configuration is illustrated in Fig. 5, in which motor, inverter, and SAZZ chopper are the identical as is used in the EV mentioned later.

The basic specifications of PMSM, inverter[21], SAZZ chopper, and Lithium-ion battery are summarized in Table II-Table V. The load torque in the motor test bench is simulated as if the actual vehicle runs, the several key characteristics of which are summarized in Table VI. The total driving resistance force is a function of friction force, aerodynamic force, and acceleration/deceleration force. The driving mode of the simulated vehicle in the laboratory is taken from "JC08 mode" driving pattern, as shown in Fig. 6. This new driving pattern test is mandatory for the new cars in sale starting April 2011 in Japan, in which the driving distance is $8.2 \mathrm{~km}$ and the driving time is $1200 \mathrm{~s}$.

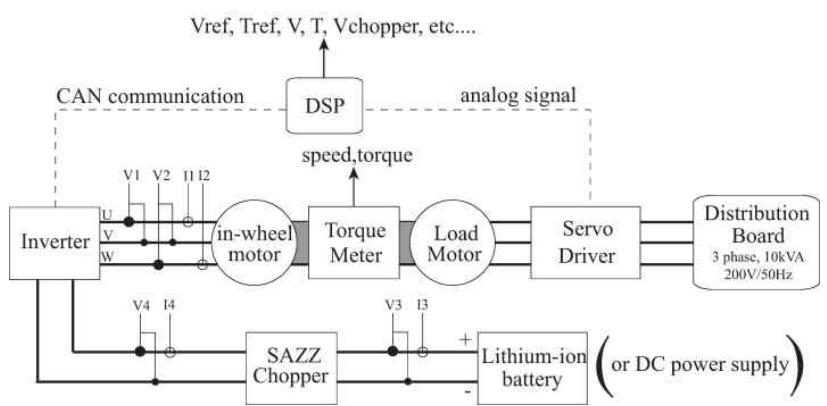

Fig. 5. Overall schematic configuration of motor test bench for evaluating EV driving performance.

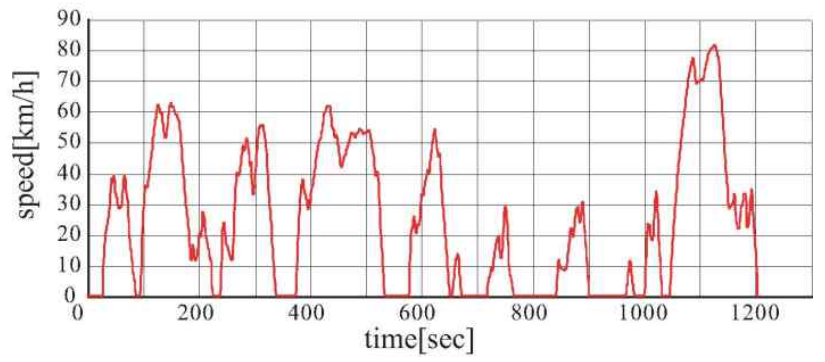

Fig. 6. "JC08 mode" driving pattern; $1200 s$ in driving time and $8.2 \mathrm{~km}$ in driving distance.

TABLE II

SPECIFICATIONS OF IN-WHEEL PMSM FOR EV DRIVE

\begin{tabular}{l|c}
\hline \multicolumn{1}{c|}{ item } & value \\
\hline \hline maximum output power & $20 \mathrm{~kW}(382 \mathrm{rpm}, 20 \mathrm{~s})$ \\
\hline maximum torque & $500 \mathrm{Nm}(20 \mathrm{~s})$ \\
\hline rated output power & $6 \mathrm{~kW}(521 \mathrm{rpm})$ \\
\hline rated torque & $110 \mathrm{Nm}$ \\
\hline rated speed & $1113 \mathrm{rpm}(115 \mathrm{~km} / \mathrm{h})$ \\
\hline
\end{tabular}

TABLE III

SPECIFICATIONS OF INVERTER

\begin{tabular}{l|c}
\hline \multicolumn{1}{c|}{ item } & information \\
\hline \hline control method & vector control \\
\hline switching frequency & $6 \mathrm{kHz}$ \\
\hline communication for signal & $\mathrm{CAN}$ \\
\hline
\end{tabular}

TABLE IV

SPECIFICATIONS OF SAZZ CHOPPER

\begin{tabular}{l|c}
\hline \multicolumn{1}{c|}{ item } & value \\
\hline \hline rated output power & $8 \mathrm{~kW}$ \\
\hline maximum output power & $25 \mathrm{~kW}(20 \mathrm{~s})$ \\
\hline rated voltage of battery & $300 V_{\mathrm{DC}}\left(240 \sim 370 V_{\mathrm{DC}}\right)$ \\
\hline rated voltage of inverter & $400 V_{\mathrm{DC}}\left(20 \sim 420 V_{\mathrm{DC}}\right)$ \\
\hline switching frequency & $25 \mathrm{kHz}$ \\
\hline dimensions $(\mathrm{W} \times \mathrm{H} \times \mathrm{D})$ & $339 \mathrm{~mm} \times 170 \mathrm{~m} \mathrm{~m} \times 350 \mathrm{~mm}$ \\
\hline
\end{tabular}


TABLE V

SPECIFICATIONS OF LITHIUM-ION BATTERY

\begin{tabular}{c|c|c}
\hline item & unit & value \\
\hline \hline total voltage & $V$ & $334 \sim 208$ \\
\hline total power & $k \mathrm{Wh}$ & 5.5 \\
\hline
\end{tabular}

TABLE VI

Specifications OF Motor Test Bench

\begin{tabular}{l|c|c|c}
\hline \multicolumn{1}{c|}{ item } & definition & unit & value \\
\hline \hline total inertia & $J_{m}+J_{L}$ & $\mathrm{~kg} \cdot \mathrm{m}^{2}$ & 0.45 \\
\hline $\begin{array}{l}\text { coefficient of total viscous } \\
\text { friction }\end{array}$ & $D_{m}+D_{L}$ & $\mathrm{~kg} \cdot \mathrm{m}^{2} / \mathrm{ms}$ & 0.065 \\
\hline gross vehicle weight & $M$ & $\mathrm{~kg}$ & 800 \\
\hline $\begin{array}{l}\text { coefficient of rolling } \\
\text { friction }\end{array}$ & $\mu$ & - & 0.00747 \\
\hline constant drag & $C_{d}$ & - & 0.23 \\
\hline tire radius & $r$ & $\mathrm{~m}$ & 0.275 \\
\hline gravitational acceleration & $g$ & $\mathrm{~m} / \mathrm{s}^{2}$ & 9.8 \\
\hline frontal projected area & $A$ & $\mathrm{~m}^{2}$ & 1.6 \\
\hline atmospheric density & $\rho$ & $\mathrm{kg} / \mathrm{m}^{3}$ & 1.23 \\
\hline
\end{tabular}

\section{B. Efficiency of Each Component at Constant Operating Point}

When the simulated vehicle runs according to "JC08 mode" driving pattern, it will go through many operating points, which are at different load torque, depending on the vehicle speed under powering and motoring mode operations. The efficiency of the inverter-motor is measured under the combination conditions of both speed and load-torque.

Fig. 7 shows one example result measured under the combination condition of both the constant load torque $(75 \mathrm{Nm})$ and the variable speed ranges $(10 \mathrm{~km} / \mathrm{h} \sim 70 \mathrm{~km} / \mathrm{h})$. As shown in this figure, it can be easily confirmed that the Quasi-PAM controlled inverter is better in the efficiency of inverter itself or inverter-motor than the Pure-PWM controlled inverter.

The chopper efficiency was also measured as the function of speed and power. In Fig. 8, the total efficiency excluding or including SAZZ chopper is analyzed for comparison between the Pure-PWM controlled inverter and the Quasi-PAM controlled inverter. In this figure, data at the left side or the right side of each operating point is analyzed under excluding or including SAZZ chopper efficiency respectively. This figure indicates firstly that the invertermotor efficiency was increased by the Quasi-PAM controlled inverter, but secondly that the total efficiency is not so much improved because the total efficiency is a multiplication of the chopper efficiency and the inverter-motor efficiency.

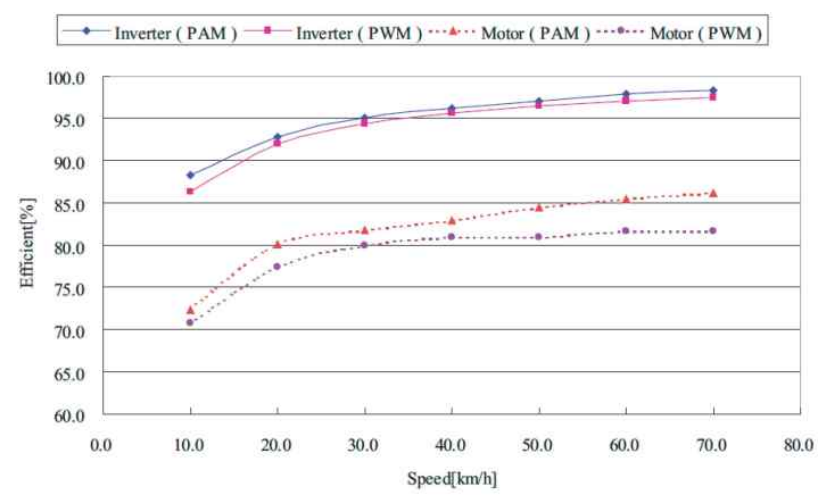

Fig. 7. One example of efficiency comparison of inverter itself or inverter-motor by Pure-PWM controlled inverter and Quasi-PAM controlled inverter in case of $75 \mathrm{Nm}$.

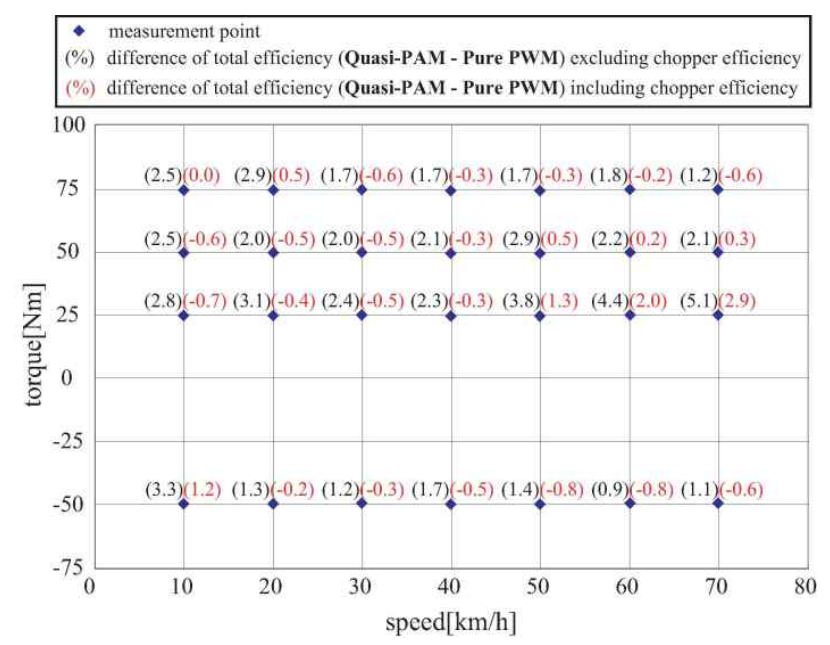

Fig. 8. Total efficiency comparison between Pure-PWM controlled inverter and Quasi-PAM controlled inverter; data at the left side or the right side of each operating point is analyzed under excluding or including SAZZ chopper efficiency respectively.

In the series chopper based EV power train, the chopper efficiency is largely dependent on the speed-torque operation region as shown in this figure. For example, in the operation regions of lower speed-torque and higher speed-torque, it is found that Quasi-PAM inverter including chopper efficiency might be slightly lower in efficiency than Pure-PWM inverter.

If the output torque is relatively small under highly efficient chopper, the total efficiency of the Quasi-PAM controlled inverter is better than that of the Pure-PWM controlled inverter. The efficiency measurement of SAZZ chopper will be shown in the next subsection IV-C with the running operating conditions.

\section{One Charge Driving Distance under Motor Test Bench}

The Lithium-ion battery is charged up to full and one charge driving distance is measured according to JC08 mode driving pattern until the battery voltage reaches the minimum allowable rated value. The driving resistance is simulated on 
the basis of Table VI. The friction resistance and the aerodynamic resistance are artificially simulated by the load motor, as well as the acceleration force and the deceleration force required for the JC08mode driving pattern.

The total energy consumption is measured by the power meter and the torque-speed meter, in which the battery output terminal, the chopper output terminal, and the inverter output terminal are measured by the power meter, and the torque and the speed are measured by the torque meter and the speed meter respectively. The driving distance is calculated by the integration of the simulated vehicle speed. The mileage measured by the motor test bench is calculated by the quotient of the driving distance $[\mathrm{km}]$ divided by the total energy consumption $[k \mathrm{Wh}]$.

The output voltage of SAZZ chopper is controlled so that it is constant at $150 \mathrm{~V}$ until the speed of $50 \mathrm{~km} / \mathrm{h}$, and then it is proportionally increased to the speed in the speed region over $50 \mathrm{~km} / \mathrm{h}$.

The battery voltage drops as the simulated vehicle runs more. The braking characteristics are shown in Fig. 9, in which the electric braking is relatively large in the low speed region and the mechanic braking increases when the speed increases.

The CEMF voltage of the motor is a function of speed. Thus the maximum inverter output voltage, which is determined by battery voltage, should be at least equal to or larger than that of the required inverter voltage. This can produce the electric torque so that the vehicle can run at the maximum speed. The battery voltage decrease as the battery SOC becomes lower. The field weakening is one technique to avoid this problem, but the motor efficiency rapidly decreases if this is applied. In this paper, it is assumed that the battery voltage cannot be reduced beyond a certain thresh hold level, because the inverter-motor drive cannot achieve the rated maximum speed. This relation is shown in Fig. 10. The battery voltage and the achievable highest speed are plotted and also the CEMF voltages are measured. Through Fig. 10, authors assume that if the battery voltage becomes below $250 \mathrm{~V}$, the maximum speed cannot be achieved without chopper. The measured battery voltage and SOC are plotted in Fig. 11.

The measured driving distances are summarized in Table VII. The driving distance by the Quasi-PAM controlled inverter is $101.2 \mathrm{~km}$, and that of the Pure-PWM controlled inverter is $94.3 \mathrm{~km}$. This means that the Quasi-PAM controlled inverter gives 7\% longer driving distance than the Pure-PWM controlled inverter. Therefore, the voltage boost capability can make the overall performance of Quasi-PAM better than that of the Pure-PWM controlled inverter. The efficiency map of SAZZ chopper under JC08 mode driving pattern is shown in Fig. 12.
TABLE VII

JC08 Mode's Driving Distance at Motor Test Bench

\begin{tabular}{l|c|c}
\hline & $\begin{array}{c}\text { Pure-PWM inverter } \\
\text { without chopper }\end{array}$ & $\begin{array}{c}\text { Quasi-PAM inverter } \\
\text { with chopper }\end{array}$ \\
\hline \hline energy consumption & $5.59 \mathrm{kWh}$ & $5.59 \mathrm{kWh}$ \\
\hline driving distance & $94.3 \mathrm{~km}$ & $101.2 \mathrm{~km}$ \\
\hline mileage & $16.9 \mathrm{~km} / \mathrm{kWh}$ & $18.1 \mathrm{~km} / \mathrm{kWh}$ \\
\hline
\end{tabular}

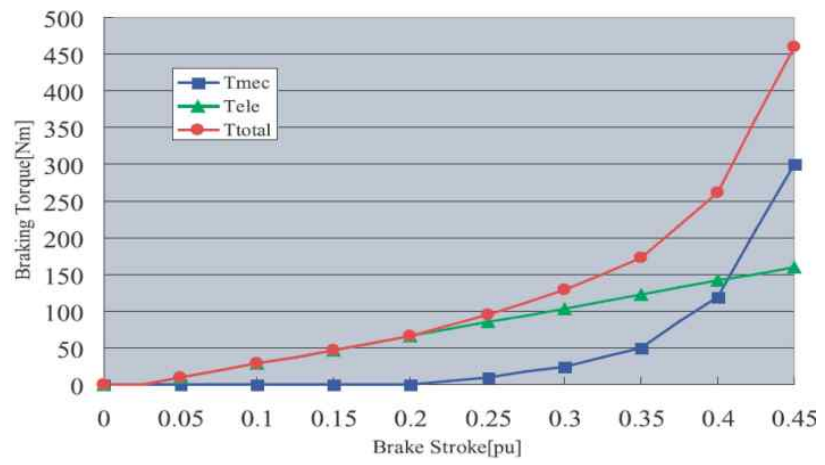

Fig. 9. Braking characteristics by relation between bake stroke and baking torque; $T_{\text {mech }}$ is mechanical braking torque, $T_{\text {elec }}$ is electric braking torque, $T_{\text {total }}$ is the sum of $T_{\text {mech }}$ and $T_{\text {elec }}$.

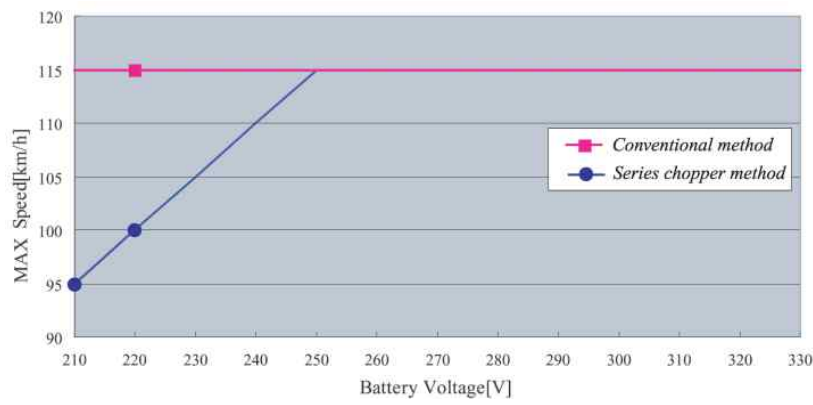

Fig. 10. Relation between battery voltage and the maximum motor speed.

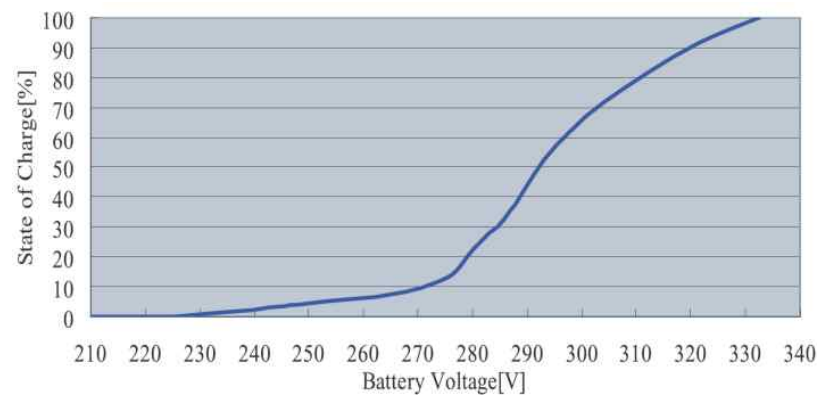

Fig. 11. Relation between output voltage and SOC in Lithium-ion battery. 


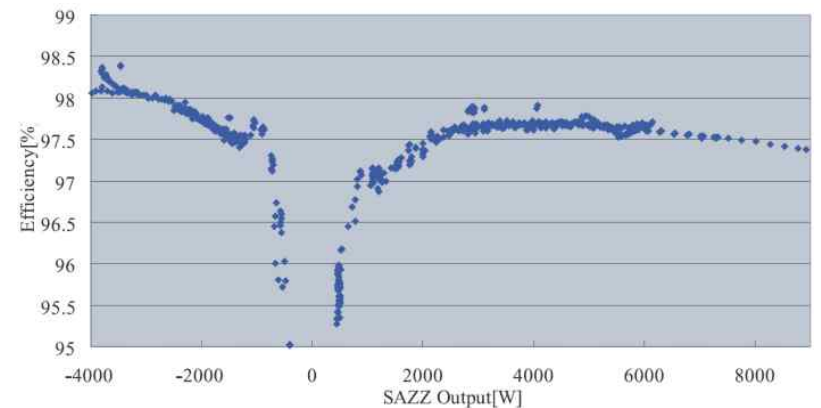

Fig. 12. Efficiency map of SAZZ chopper measured under JC08 mode driving pattern.

\section{CHASSIS DYNAMO BASED DRIVING PERFORMANCE EXPERIMENTAL ANALYSIS}

The two sets of chopper, inverter-motor, and battery mentioned in the previous section are actually installed in the prototype electric vehicle called as KAST-EV "KANA". This vehicle is tested under the chassis dynamo experimental facility, and the driving distance is measured on the basis of JC08 mode driving pattern. The chassis dynamometer used in this paper is a vehicle's performance-evaluation facility by measuring the power delivered to the surface of the "drive roller" by the drive wheels. The vehicle is often parked on the roller or rollers, which the vehicle then turns and the output is measured.

In this experiment, the vehicle weight is adjusted depending on whether the SAZZ chopper of $38.2 \mathrm{~kg}$ is on board or not. As a result, the friction lag force and acceleration force were reflected this time.

The driving distance is summarized in Table VIII, in which three cases are compared: (a) Pure-PWM without chopper, (b) Quasi-PAM with chopper, and (c) Quasi-PAM with $1 / 2$ weight chopper. The chassis dynamo test was executed only 5 times, due to the limited budget and the trouble shootings.

This table indicates that the series chopper based EV power train may be useful at the present weight of $38.2 \mathrm{~kg}$. However, two further investigations are required: (1) the test should be repeated for securing the reliable data, (2) the chopper weight has a large effect on the mileage, (3) the motor bench test results in the previous section has a good agreement to this result, because the chopper weight is not considered under that test in the previous section.

The following suggestion can be observed in this paper: the series chopper power train for EV is useful in order to extend the driving distance if the high efficiency and the low weight of chopper are satisfied.

\section{CONCLUSION}

In this paper, the series chopper based power train for EV was proposed and the higher efficiency for EV drives can be expected compared with the chopperless typed power train. To verify the proposed concept for EV, the motor test bench was constructed in the laboratory and the chassis dynamo was
TABLE VIII

Driving Distance of Chassis Dynamo BASED on JAPANESE Official Driving Test Regulation (JC08 Mode) (Tested AT MAy 22-27, 2011)

\begin{tabular}{|c|c|c|}
\hline type of EV power train & driving distance & ratio \\
\hline Pure-PWM without chopper ${ }^{1)}$ & $85.6 \mathrm{~km}$ & $100 \%$ \\
\hline Quasi-PAM with chopper ${ }^{2)}$ & $85.8 \mathrm{~km}$ & $100.2 \%$ \\
\hline Quasi-PAM with $1 / 2$ weight chopper ${ }^{3)}$ & $88.2 \mathrm{~km}$ & $103.0 \%$ \\
\hline
\end{tabular}

used in the specific driving performance test facility. It is confirmed through two kinds of experimental analysis that the series chopper based EV power train has the better driving performance under satisfying the high efficiency and the low weight of chopper.

The inverter-motor efficiency was improved by the Quasi-PAM controlled inverter, but the total efficiency with SAZZ chopper is a little less than that of Pure-PWM controlled inverter.

The tentative chassis dynamo experiments indicate that the series chopper power train, which has SAZZ chopper with average efficiency $97.5 \%$ and weight $38.2 \mathrm{~kg}$, has a little longer driving distance compared with the Pure-PWM scheme. If the chopper weight becomes half, $3 \%$ better efficiency was obtained.

\section{ACKNOWLEDGMENT}

This project was partially supported by the KAST (Kanagawa Academy of Science and Technology).

\section{REFERENCES}

[1] H. Shimizu, To Protect Global Warming, Random House Publisher, Japan, 2007.

[2] Y. Hori, "Application of advanced motion control techniques and the prospect of super capacitors in electric vehicles," Proceedings of AMC, 2006.

[3] T. Hosokawa, K. Tanihata, and H. Miyamoto, "Development of iMiEV next generation," Mitsubishi Motors Technical Review (2nd Report), No. 20, pp. 52-59, 2008.

[4] TRIAS 5-10-2008.

[5] A. Kawamura, Y. Tsuruta, and S. Inasaka, "Proposal of denpi for the commuter vehicle and the discussion," Proceedings of IEEJ VT-07-13, 2007.

[6] S. Inasaka, Y. Tsuruta, and A. Kawamura, "Discussion on high efficient DC power management for EVs," Proceedings of Korea-Japan Technical Workshop on SPC, pp. 67-70, 2008.

[7] Y. Tsuruta, Y. Ito, and A. Kawamura, "A new zerovoltage-zero-current-transition chopper SAZZ for a FCEV drive," Proceedings of PESC, Raleigh, USA, 2005.

[8] Y. Tsuruta, M. Bando, Y. Ito, A. Kawamura, "A new circuit geometry sazz for an ev drive application," Proceedings of IAS, pp. 2144-2149, 2006.

[9] Y. Tsuruta, Y. Ito, and A. Kawamura, "Snubber assisted zero voltage and zero current transition bilateral buck and 
boost chopper for ev drive application and test evaluation at 25kW," IEEE Trans. Ind. Electron., Vol. IE-56, No.1, pp.361-371, Jan. 2009.

[10] M. Pavlovsky, Y. Tsuruta, and A. Kawamura, "Recent improvement of efficiency and power density of DC-DC converters for automotive applications," Proceedings of IPEC-Sapporo, pp.1866-1873, 2010.

[11] Y. Tsuruta, M. Pavlovsky, G. Guidi, and A. Kawamura, "Four quadrant SAZZ-1 chopper for EV and HEV power train," Proceedings of ICPE and ECCE Asia, pp. 1016-1023, 2011.

[12] A. Kawamura, T. Yokoyama, H. Funato, N. Hoshi, and Yoshino, Introduction to Power Electronics, Corona Publisher, 2009.

[13] S. Tsutsuki, Y. Watanabe, Y. Tsuruta, R. Kamei, G. Giuseppe, and A. Kawamura, "Experimental data analysis on total driving performance of series chopper based EV power train," Proceedings of IEEJ VT-11-09, Jan. 2011.

[14] A. Kawamura, G. Guidi, S. Tsutsuki, Y. Watanabe, Y. Tsuruta, and N. Motoi, "Experimental data analysis on total driving performance of series chopper based EV power train," Proceedings of IECON, pp. ,2011.

[15] Y. Watanabe, G. Guidi, and A. Kawamura, "Motor efficiency improvement case study of the minimum total harmonic distortion pulse amplitude control method," Proceedings of JIASC2011, pp. 649-652, 2011.

[16] R. G. Hoft, Semiconductor Power Electronics, Van Nostrand Reinhold, 1986.

[17] Y. Takeda, N. Matsui, S. Moromoto, and Y. Honda, IPM Motor-Seginn and Control, Ohm Publisher, 2001.

[18] N. Takahashi, "Advanced electromagnetic field analysis and its application to electrical machines," IEEJ Trans. on IAS, Vol. 123, No. 4, pp.317-322, 2003.

[19] L. Gao, S. Liu, and R. A. Dougal, "Dynamic lithium-ion battery model for system simulation," IEEE Trans. Comput.Packag., Vol. 25, No. 3, pp. 495-505, Sep. 2002.

[20] M. Chen and G. A. Rincon-Mora, "Accurate electrical battery model capable of predicting runtime and I-V performance," IEEE Trans. Energy Convers., Vol. 21, No. 2, pp.504-510, Jun. 2006.

[21] Okamoto, Kiriya, and Noda, "Development of 20 inch in-wheel motor system," Toyo Denki Review, No.117, pp.12-17, 2007.

[22] J. H. Seo, T. K. Chung, S. Y. Jung, C. G. Lee, and H. K. Jung, "Harmonic iron loss analysis of permanent magnet motor for high-speed train," Journal of The Korean Society for Railway, Vol.12, No.3, pp.335-341, Jun. 2009.

[23] K. Yamazaki, "Torque and efficiency calculation of an interior permanent magnet motor considering harmonic iron losses of both the stator and rotor," IEEE Trans. Magn., Vol. 39, No. 3, pp. 1460-1463, May 2003.

[24] H. Domeki, Y. Ishihara, C. Kaido, Y. Kawase, S. Kitamura, T. Shimomura, N. Takahashi, T. Yamada, and K. Yamazaki, "Investigation of benchmark model for estimating iron loss in rotating machine," IEEE Trans. Magn., Vol. 40, No. 2, pp. 794-797, Mar. 2004.

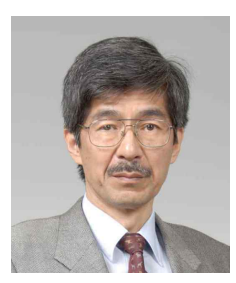

Atsuo Kawamura received the Ph.D. degrees in electrical engineering from the University of Tokyo in 1981. After the five year stay at the University of MissouriColumbia as a faculty member, he joined Yokohama National University in 1986, and now he is a professor there. His interests are in the fields of power electronics, digital control, electric vehicles, and biped robotics. He received Transactions Paper Awards from IEEE in 1988, 2001 and 2002, also from IEE of Japan in 1996.Dr. Kawamura is an IEEE Fellow, and a senior member of the IEE of Japan. He serves as a president of IEEJ/IAS starting May 2012.

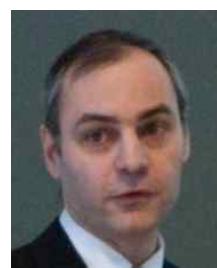

Giuseppe Guidi graduated from the University of L'Aquila, Italy, in 1995, and received his $\mathrm{Ph} . \mathrm{D}$. from the Norwegian University of Science and Technology (NTNU) in 2009. He has worked for industry in the field of Power Electronics from 1997 to 2004, joining first Fuji Electric R\&D, Japan, as R\&D engineer and then SIEI SpA, Italy as senior engineer. In 2009, he joined Yokohama National University as Research Associate. Since 2011, he is also part-time research associate with NTNU, Norway. His research interests include power electronics, traction control and drive systems for electric propulsion, as well as application of power electronics to renewable energy. Dr. Guidi is a member of the IEEE and of the IEE of Japan.

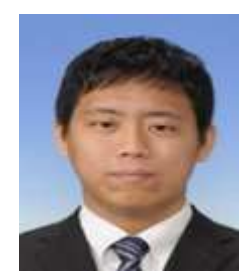

Yuki Watanabe received the B.E. degree and the M.E. degree in electrical and computer engineering from Yokohama National University, Japan, in 2010 and 2012, respectively. He currently works at DENSO Corporation.

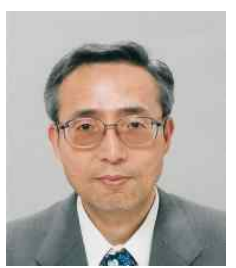

Yukinori Tsuruta received the B.S., M.S., and $\mathrm{Ph} . \mathrm{D}$. degrees in electrical engineering from Yokohama National University, Yokohama, Japan, in 1975, 1977, and 2006, respectively. From 1977 to 2003, he had been with Toshiba Corporation, Tokyo, Japan, working with the design and development section of power converter equipment as a specialist engineer in the Fuchu Works. He is currently with Yokohama National University as a postdoctoral fellow and a non-full time lecturer. His research interests include soft-switching converter systems for electric vehicle applications. $\mathrm{He}$ is a member of the IEEE and the IEE of Japan.

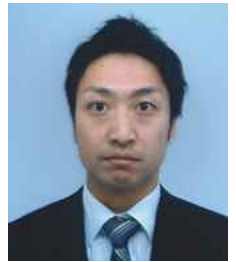

Naoki Motoi received the B.E. degree in system design engineering and the M.E. and Ph.D. degrees in integrated design engineering from Keio University, Japan, in 2005, 2007 and 2010, respectively. From 2007 to 2011, he had worked in Partner Robot Div., Toyota Motor Corporation, Aichi, Japan. He is currently a research associate at Yokohama National University. He is a member of the IEEE. His research interests include robotics, motion control, and power electronics.

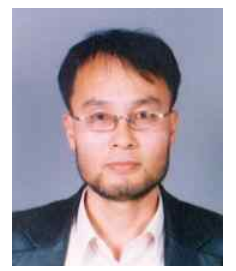

Tae-Woong Kim received his B.S. and M.S. in Electrical Engineering from Chungbuk National University, Chungbuk, Korea, in 1990 and 1993, respectively. He received his Ph.D. from Yokohama National University, Japan, in 1996. He joined the Yaskawa Electric Corporation, Japan, from April 1996 to September 2001. He was a Research 
Associate Professor for one yearat Aalborg University, Denmark. Later, he joined the faculty of Gyeongsang National University, Jinju, Korea, in October 2002, where he is presently an Associate Professor in the Department of Control and Instrumentation Engineering. His research interests include power electronics, motion control, electric vehicles, renewable energy, and applied embedded control systems. Dr. Kim is an active member ofKIPE, IEEE, IEEJ, and GNU-ERI. He received the IEEE IE Transaction Best Paper Award in 2000 and the outstanding academic co-work project award from LG Electronics DAC Lab in 2008. 